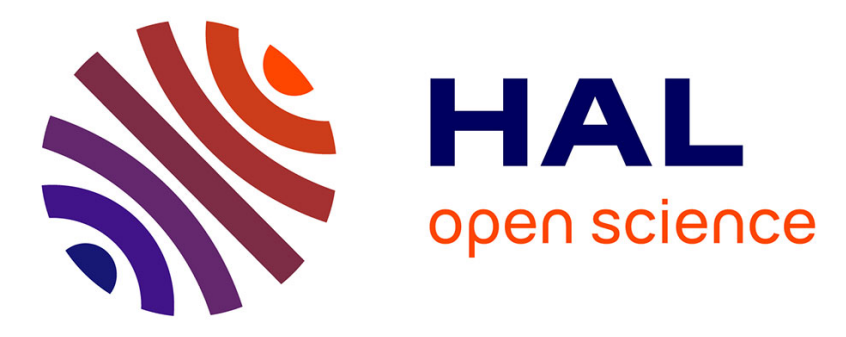

\title{
Mutational analysis-inspired algorithms for cells self-organization towards a dynamic under viability constraints
}

\author{
Alexandra Fronville, Abdoulaye Sarr, Pascal Ballet, Vincent Rodin
}

\section{To cite this version:}

Alexandra Fronville, Abdoulaye Sarr, Pascal Ballet, Vincent Rodin. Mutational analysis-inspired algorithms for cells self-organization towards a dynamic under viability constraints. Self-Adaptive and Self-Organizing Systems (SASO), 2012 IEEE Sixth International Conference on, Sep 2012, Lyon, France. pp.181-186, 10.1109/SASO.2012.15 . hal-00781080

\section{HAL Id: hal-00781080 \\ https://hal.science/hal-00781080}

Submitted on 28 Jan 2013

HAL is a multi-disciplinary open access archive for the deposit and dissemination of scientific research documents, whether they are published or not. The documents may come from teaching and research institutions in France or abroad, or from public or private research centers.
L'archive ouverte pluridisciplinaire $\mathbf{H A L}$, est destinée au dépôt et à la diffusion de documents scientifiques de niveau recherche, publiés ou non, émanant des établissements d'enseignement et de recherche français ou étrangers, des laboratoires publics ou privés. 


\title{
Mutational analysis-inspired algorithms for cells self-organization Towards a dynamic under viability constraints
}

\author{
Alexandra FRONVILLE* ${ }^{* \ddagger}$, Abdoulaye SARR ${ }^{\dagger}$, Pascal Ballet*, Vincent RODIN* \\ *European University of Britany, UBO, Lab-STICC UMR-6285-CNRS, 29238 Brest Cedex 3, France \\ ${ }^{\dagger}$ Computer Science Department, Telecom Bretagne, 29280 Plouzané, France \\ ${ }^{\ddagger}$ Corresponding author email : alexandra.fronville@univ-brest.fr
}

\begin{abstract}
In biology, recent techniques in confocal microscopy have produced experimental data which highlights the importance of cellular dynamics in the evolution of biological shapes. Thus, to understand the mechanisms underlying the morphogenesis of multi-cellular organisms, we study this cellular dynamic system in terms of its properties: cell multiplication, cell migration, and apoptosis. Besides, understanding the convergence of the system toward a stable form, involves local interactions between cells. Indeed, the way that cells selforganize through these interactions determines the resulting form. Along with the mechanisms of convergence highlighted above, the dynamic system also undergoes controls established by the nature on the organisms growth. Hence, to let the system viable, the global behavior of cells has to be assessed at every state of their developement and must satisfy the constraints. Otherwise, the whole system self-adapts in regard to its global behavior. Thus, we must be able to formalize in a proper metric space a metaphor of cell dynamics in order to find conditions (decisions, states) that would make cells to self-organize and in which cells self-adapt so as to always satisfy operational constraints (such as those induced by the tissue or the use of resources). Therefore, the main point remains to find conditions in which the system is viable and maintains its shape while renewing. The aim of this paper is to explain the mathematical foundations of this work and describe a simulation tool to study the morphogenesis of a virtual organism.
\end{abstract}

Keywords-morphological analysis; virtual reality; biological multi-agent system; mathematical programming;

\section{INTRODUCTION}

Biomedical science has undergone a remarkable evolution during this last decade. Advances and innovations in biotechnology, more particularly in microscopy and imaging, have provided a large amount of data. In 2007, Melani and al. achieved a tracking of cell nuclei and the identification of cell divisions in live zebrafish embryos using 3D+time images acquired by confocal laser scanning microscopy [1]. These data allowed new description in details of many components and structures of living organisms. So, we have looked forward to learn more about the emergent properties of cells organization and especially the importance of cellular dynamics in the evolution of shapes, both in mathematical and numerical point of view.

Complex systems can be defined as a composition of a significant number of elements interacting locally to produce a global behavior. Considering this definition, we can first consider the correspondences between the principles of artificial systems and of complex models seen in nature. These models (ripples in sand dunes, spots on animal coats, geometric figures in plants) present fascinating aspects which are the result of a process. All the laws, conditions and states of this process define the morphogenesis. According to Doursat [2], whether considering inanimate structures or living organisms, the process of morphogenesis shows how a collective self-organization aims to reach a determined shape. In this regard, Hogeweg and Marée proposed an extension of the Glazier and Graner model formalism to show how individual amoebaes aggregate to form a stalk using a sticking power mechanism [3].

Another level of analysis opens the huge possibilities created by new properties of computer systems. Indeed, biological and multi-agents system share certain characteristics such as robustness, emergence, self-organization and adaptability [4]. Used in the field of living matter, these properties lead to an increasing need of understanding, prediction and control of their functioning.

However, there is no theory proving that the behavior of these systems can be deducted from the behavior of its components, all the more so as they are characterized by a high degree of decentralization and self-organization. And most of the complex systems have a capacity of adaptation, evolving and learning through feedbacks between their external environment and their internal architecture. When cells evolve, they modify their organism which in its turn impacts its environment; in return, this latter modifies back the cells. Epigenetics considers this coupling between organism and environment and can not be ignored in understanding the development of living [5]. The cells' mechanisms of self-adaptation (top-down) and self-organization (bottom-up) through these three levels must be studied and formalized properly to understand the convergence of the system to a stable form.

Where most of the studies are only interested in global behavior of systems converging to a form, in our model every cell has its own rules controled by a set of parameters, controls and by its neighborhood. Thanks to this way, the integration of the behaviors of each component of the system allows to determine its global state. 
In mathematics, the viability theory [6] offers concepts and methods to control a dynamical system in a given fixed environment, in order to maintain it in a set of constraints of viability. But this theory is not suitable to study a cell system that grows and multiplies. Thus, a new approach stemming from viability theory, extended to multivalued analysis, gives new avenues: the mutational analysis [7].

So, the concept of differential equations has been extended to the concept of mutational equations in a metric space. In fact, morphological equations, special type of mutational equation, have similar properties to differential equations (Peano theorem, Cauchy-Lipschitz, Nagumo) [8]. They govern the evolution of sets in the same way that differential equations govern the evolution vectors.

When the system evolves the environment changes. And these changes raise some self-organization mechanisms within cells to adapt to the new environment. This is what biologists mean by co-evolution. Mathematically, it is the joint evolution of states and sets in which self-adaptations of cells have to be led. In this case, the environments are changing under the action of a morphological equation, evolutionary systems are governing the evolution of the states and of the environment, and they depend on both the state and the environment. This is called a differentialmorphological system. For such a differential-morphological system to have solutions we have to adapt the viability theorem to the differential-morphological systems. This means that there is at least one co-viable evolution of the state and the environment based on each state-environment pair. The set of conditions for which at least one solution is viable is called viability kernel. Regards to this definition, the viability kernel implies a question of determining the time when the state of the cells reaches its limits of viability.

Thinking of morphogenesis in this way brings new requirements, particularly in mathematics and computer science to implement efficient mutational algorithms able to inform us about self-organization and self-adaptation mechanisms involved in multi-cellular organisms survival. Besides, the definition of the viability kernel remains difficult: specific algorithms have been developed however their application requires an exponential memory space with the dimension of space, and the outcome is difficult to handle.

In this paper, we are going to formalize mathematically a model of cell dynamic on the principles of morphological analysis and to describe a simulation tool for studying morphogenesis of virtual multi-cellular organisms. Morphological analysis and viability theory are the mathematical foundations of this work and the tool presented will test whether a system generated by morphological equations can self-organize to maintain its shape and self-adapt to remain "viable" in a given environment constraints.

\section{MoRPhOlOGICAL DYNAMiC OF CELlULAR TISSUE} EVOLUTION

The purpose of this paragraph is to formalize in the context of mutational and morphological analysis [7], [8], the evolution of cellular tissues during embryogenesis. It provides an extension of differential equations in a metric space instead of the classical Euclidean space $\mathbb{R}^{N}$. This question motivates the study of a discrete morphological dynamical system governing the evolution of tissues.

At the tissue level, we have a large group of connected cells of the same type performing a specific function [9]. Therefore, the functionning and evolution of cellular tissue can be seen as a result of a bottom-up mechanism of cellular dynamic. An infinitesimal change of tissue implies a selforganization of that dynamic where each element of the form is not only "move" to another point of the form that follows it, but eventually moved and "multiplied" when multiple daughter cells succeed to this element, multivalent character which leads to the concept of speed form [7].

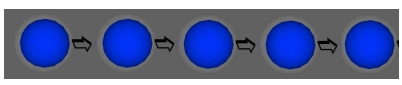

Figure 1. Univalued analysis to formalize a cell that moves

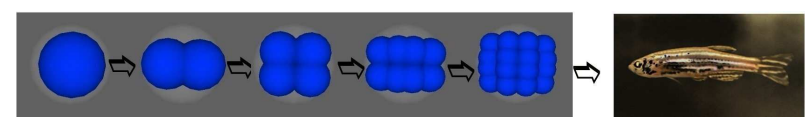

Figure 2. Multivalued analysis to formalize a cell that multiplies and moves

During embryonic development, the confinement is imposed by the cohesion of tissues and the presence of an envelope, such as the epithelial layer covering the embryo. There is a co-evolution of the cellular membrane and the dynamics of each cell, confinement shapes that can evolve only by respecting the constraints that we want to study using morphological analysis. This co-evolution is only possible through top-down mechanisms by which cells self-adapt their dynamic with respect to the constraints.

In biological morphogenesis, the vitellus is the energy reserves used by the embryos during embryonic development. $M$ denotes the set of containment cells, contained in the complement of vitellius.

$K \subset \mathbb{R}^{3}$ representing tissue cells, the cells are designated by $x \in K \subset \mathbb{R}^{3}$.

If we restrict morphogenesis in the plan,

$$
\mathcal{D}:=\{(1,0),(-1.0)(0,1),(0,-1)\}
$$

or shortly

$$
\mathcal{D}:=\{1,-1,2,-2\}
$$

denotes the set of 4 planes directions and

$$
\overline{\mathcal{D}}:=\mathcal{D} \cup\{(0,0)\} \cup \emptyset
$$


means the 6 "extended" directions

For morphogenesis in the space $\mathbb{R}^{3}$,

$$
\begin{gathered}
\mathcal{D}:=\{(1,0,0),(-1,0,0),(0,1,0),(0,-1,0),(0,0,1), \\
(0,0,-1)\}
\end{gathered}
$$

or shortly

$$
\mathcal{D}:=\{1,-1,2,-2,3,-3\}
$$

denotes the set of six directions and $\overline{\mathcal{D}}:=\mathcal{D} \cup\{(0,0,0)\} \cup \emptyset$ means the eight "extended" directions.

We denote by $A+\emptyset=\emptyset$ in the max-plus algebra for the operations $\cup$ and + .

We will note

$$
\Xi_{M}(K, x):=\{u \in \mathcal{D} \text { such that } x+u \in\{x\} \cup(M \backslash K)\}
$$

and

$$
R_{M}(K, x):=\Xi_{M}(K, x) \times \Xi_{M}(K, x) .
$$

Then we introduce the correspondence

$$
\Psi(x, u, v):=\{x+u\} \cup\{x+v\}_{(u, v) \in R_{M}(K, x)} .
$$

The morphological dynamic $\Phi_{M}$ is then defined by

$$
\Phi_{M}(K):=\bigcup_{x \in K} \bigcup_{(u, v) \in R_{M}(K, x)} \Psi(x, u, v)
$$

And the discrete morphological dynamic $K_{n+1}=\Phi_{M}\left(K_{n}\right)$. This gives the different cases of cell behavior:

1) apoptosis, obtained by taking $(\emptyset, \emptyset) \in R_{M}(K, x)$ since $\Psi(x, \emptyset, \emptyset):=\emptyset \cup \emptyset=\emptyset$

2) migration by taking $u \in \mathcal{D}$ et $v=\emptyset$ or $u=\emptyset$ and $v \in \mathcal{D}$ or further $u=v$

3) stationarity, which is a migration obtained by taking $u$ and $v$ equal to $(0,0,0)$

4) cell division by taking $u:=(0,0,0)$ et $v \in \Xi_{M}(K, x)$ (or otherwise)

5) division and migration by taking $u \in \Xi_{M}(K, x)$ and $v \in \Xi_{M}(K, x)$

We can now introduce the equivalence relation on the directions

$$
u \equiv_{x} v \text { if and only if } x+u=x+v
$$

which we denote by $\mu$ and $\nu$ the representatives, noting that by construction, for every pair $(\mu, \nu)$ the equivalence class, for all $u \in \mu$ and $v \in \nu, \Psi(x, \mu, \nu)=\Psi(x, u, v)$ does not depend on the choice of directions belonging to equivalence classes.

Because two cells can not occupy the same position, we select at most one extensive direction in each class.

The correspondence of regulation is defined by the quotient set :

$$
\Theta_{M}(K, x):=R_{M}(K, x) / \equiv_{x}
$$

The morphological dynamic $\Phi_{M}$ is always defined by

$$
\begin{aligned}
\Phi_{M}(K) & :=\bigcup_{x \in K(\mu, \nu)) \in \Theta_{M}(K, x)} \Psi(x, \mu, \nu) \\
& =\bigcup_{x \in K} \bigcup_{(u, v) \in \in R_{M}(K, x)} \Psi(x, u, v)
\end{aligned}
$$

In the case of a discrete dynamic, it is defined by control sequences $\left(u_{n}, v_{n}\right)$ associated to $K_{n}$ to define $K_{(n+1)}$. Implementation of the algorithm is equivalent to setting the viable directions.

\section{SEGMENTATION IN MORPHOGENESIS}

Here is an example of how we code the first segmentations in this exemple

$\forall x \in K_{1}=\{(0,0,0)\}$, the first route choice is $U(1, x)=$ $U(1)=[1,-1,2,-2,3,-3,0]$ will be used for the first step (see figure 3):
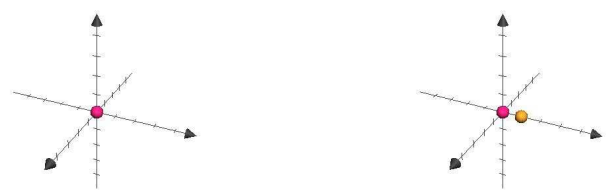

Figure 3. $U(1, x)=U(1)=[1,-1,2,-2,3,-3,0]$ means that the first axis of segmentation is $x$-axis and the direction is +1

$\forall x \in K_{2}=\{(0,0,0),(1,0,0)\}$, the second route choice is

$$
U(2, x)=U(2)=[2,-2,1,-1,3,-3,0]
$$

will be used for the second step (see figure 4) :
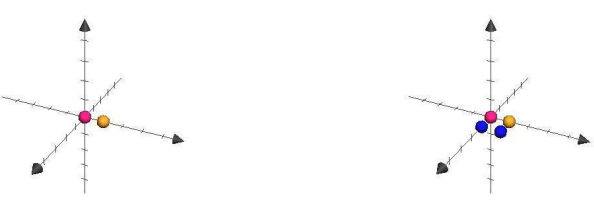

Figure 4. $U(2, x)=U(2)=[2,-2,1,-1,3,-3,0]$ means that the second axis of segmentation is $y-$ axis and the direction is +2

$\forall x \in K_{3}=\{(0,0,0),(1,0,0),(0,1,0),(1,1,0)\}$, the second route choice is

$$
U(3, x)=U(3)=U(1)=[1,-1,2,-2,3,-3,0]
$$

will be used for the third step (see figure 5) :

$\forall x \in K_{4}=\{(0,0,0),(1,0,0),(0,1,0),(1,1,0),(0,-1,0)$, $(0,2,0),(1,-1,0),(1,2,0)\}$, 

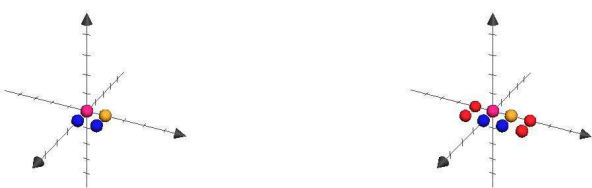

Figure 5. $U(3, x)=[1,-1,2,-2,3,-3,0]$ means that the third axis of segmentation is $x-$ axis and the direction is +1 or -1 in second choice

the fourth route choice is

$$
U(4, x)=U(4)=U(2)=[2,-2,1,-1,3,-3,0]
$$

will be used for the fourth step.

For the following step, the route choice can be choose in the list of all possibles route choice.

In a second step, for this direction choice, we have arbitrary choose the first free place in the sequence of empty space.

$$
v \in \Xi_{M}\left(K_{n}, x\right)=\left\{v \mid x+v \in M \backslash K_{n}\right\}
$$

The list of directions in $V(n, x)$ for which, the place is empty in $\left(M \backslash K_{n}\right)$ is $R(n, x)$

Then for each $x \in K_{n}$, we have a direction $v \in R(n, x)$. And $\psi(x, 0, v)=\{x\} \cup\{x+v\}$

$$
\begin{aligned}
K_{n+1}=\Phi_{M}\left(K_{n}\right) & =\bigcup_{\substack{x \in K_{n} \\
v \in R(n, x)}} \Psi(x, v) \\
& =\bigcup_{x \in K_{n}} \Psi(x, R(n, x))
\end{aligned}
$$

\section{FIRST STEPS FOR A DICTIONARY OF SHAPES}

Using this formalisation, we are starting a dictionary of reachable shapes. This dictionary will help to understand how organisms maintain their shape and which controls are used by life. The specific form of some organisms could give some ideas about life controls.

For example, in $2 \mathrm{~d}$ proliferation, the sequence is arbitrary choosen and the choice of direction is encode like $\{V(1), V(2), V(2), V(2), V(2)\}$, for different code, we get the shapes represented in figure 6 .

We have to complete now this dictionary (in $2 \mathrm{~d}, 3 \mathrm{~d}$, changing the order of browsing in a list of cells or changing the choice of mitosis direction with time ... ), it will be a help to find the encodage of shapes and to understand the link between shapes formation and cells proliferation. The mutational analysis will permit to find, for a given shape, the possible encodages.

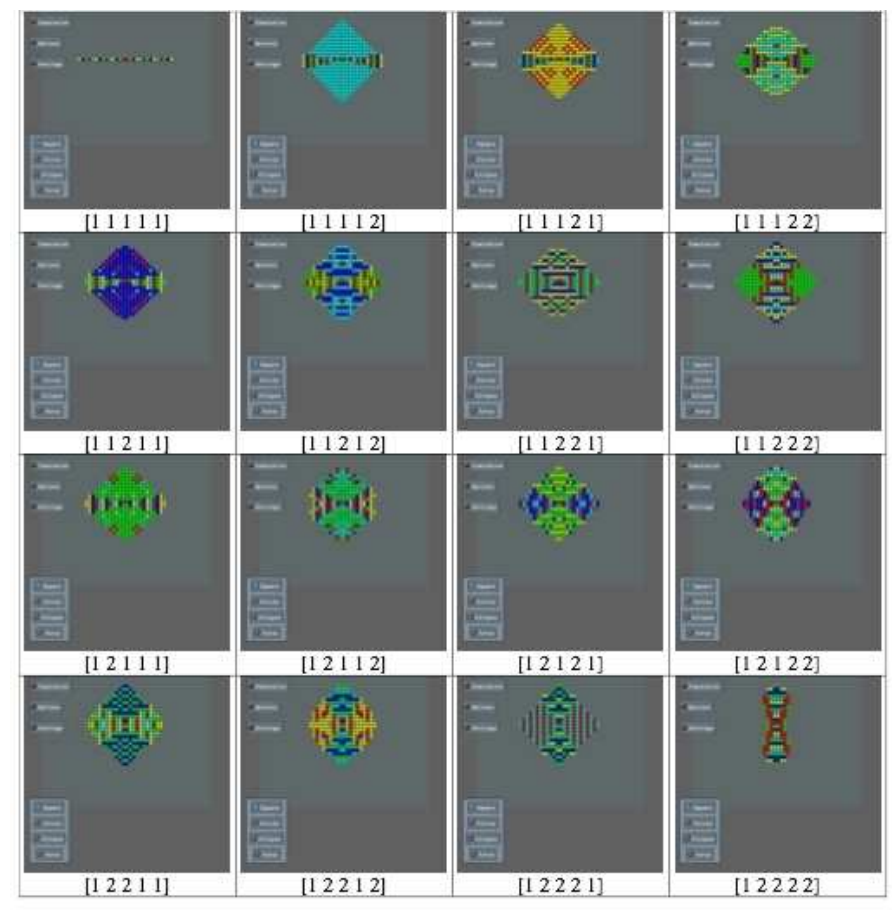

Figure 6. Dictionary of shapes

\section{TOOL FOR MODELLING MORPHOGENETIC BEHAVIOUR}

This section presents a tool developed for modelling morphogenetic phenomena by using the theorical formalism we have seen previously.

We set as a basic principle that cells are autonomous agents. A cell perceives changes in the environment and can change and adapt its dynamic accordingly.

In addition, the cells are autonomous by ignorance of the whole system because the reductionist method does not predict the evolution of the whole system. The principle that cells must be autonomous is set as a basic rule.

The platform was created to understand morphogenesis as the theoretical basis for morphological analysis. The program is implemented (applied) in $\mathrm{C}++$ using a toolkit of Virtual Reality AREVI [10], [11], it is a simulation library of autonomous entities and 3D rendering.

The order of scheduling has a significant impact on the results of the simulation [12], [13]. It determines how local interactions (self-organization) have been held, consequently how and when to get the global state of the system. Different behaviours can be observed in virtual models depending on the order of the scheduler. In nature, morphogenesis show us robust forms despite autonomous cells. To better appreciate and understand the mechanisms that are implied in morphogenesis, we wanted to retain flexibility in the scheduling of cells. The program has two different modes of simulation, a stochastic mode and a controlled mode. The cells represented on screen by spheres can proliferate 
in a discrete environment (cellular automaton) or in a "continuous" one. In the latter case the movement of cells is more precisely described and we can have more complex interactions.

Cell's behavior is not the same in each type of simulation. A simple graphical interface has been implemented in order to select the features of the simulation. It allows dynamic change of parameters and selecting mechanisms (e.g. apoptosis, differentiation) that are active/inactive during the simulation. A number of parameters were taken into account to evaluate their relative influence on the shapes generated by populations of cells.

Options are available to allow choice between 2D/3D, discrete or continuous simulations. The size and shape of both the environment and the cells can also be defined and adjusted, as can cell behaviour such as apoptosis, the direction of mitosis etc.

In the case of continuous simulation, each cell can perceive its neighbors within a radius of attraction and evaluate the stresses:

- by the neighbors.

- by the membrane containment.

Constraints are crucial for evolution of the cell; if they are too strong, the cell is not viable as it can no longer divide. A maximal constraint parameter sets up a threshold below which the cell remains viable.

The notion of coercion has no place when cells are represented in a grid. For taking into account the influence of the environment, a parameter is defined as the maximum number of cells that a cell is able to displace when it divides itself. When the number of cells is greater than the maximum stress threshold, the cell can no longer divide. To stay alive, cell can undertake two modes of mitosis which can be seen as its adaptation mechanism to face spatial constraints. Firstly, the cell chooses to divide in the direction where the stress is less intense; secondly where the direction of cell division is predetermined. However in both cases, if the spatial constraints of the current cell exceed the maximum stress threshold, it cannot divide.

It is also possible to assign an amount of energy to each cell. The basic idea is: consider that a cell has a store of energy assimilate from its environment. A percentage of the store is used to maintain structure and growth. The remaining its reserve is used for maturation (e.g. maintenance of the immune system) and reproduction. In very simple terms initially we want to apply this principle. A level of energy is mapped and associated with virtual cell application. A small amount of energy representing cell maintainance of its structure is logged at each step of the simulation. We consider that a cell uses a lot of energy in reproduction - during mitosis this energy level is divided by two. The cell dies when the energy level becomes too low. The behaviour of a cell is directly related to the quantity of energy contained and it is possible to obtain forms of very different population of cells by modulating certain thresholds, as detailed below. A cell can recover energy if it is in contact with a relevant part of the environment.

An option of the application allows cells to differentiate. In this case, cells that are not the same type (represented by different colors) have different dynamics. A specific cell can differentiate when under stress. We wanted to demonstrate this in connection with the spatial constraints of the cells. Stress corresponds to a large differential spatial constraint between two consecutive measurements. A threshold defines the minimum value of the interval for which the cell differentiates. It is also possible to define a numerical value for stress necessary to induce differentiation.

Another control is the direction of cell division. It is possible to define the direction that mitotic cells take in advance and the order of selection. This parameter can also be chosen as a random option. The morphogenesis changes when varying the choice of these directions. Real time morphogenesis film has produced data that demonstrates the features of the direction of cell division.

Parameter values modulate cells activity and the way they self-organize. The steps of the algorithm define cell behaviour and are the same for both discrete and continuous cases, as we have seen, the stress calculations are different.

\section{Evaluation}

As described in the preceding paragraph, by varying the parameters the application offers the possibility to make different types of simulation.

In collaboration with Nadine Peyrieras [1], we compared the behaviour of our model with the first segmentation of zebrafish cells. (See Figure 7).

The model shows the first segmentation of the small fish up to 1,000 cells, then the model cannot be used further because the dynamic of certain cells has changed. To enable biologists to continue to advance understanding on the establishment of the dorsoventral axis of the zebrafish, it is important to elucidate the cellular dynamics.

This question leads us to examine the outcome of differentiated cells. This motivated the development of morphological analysis to control cell dynamics and the creation of a simulation platform to visualize and compare with biological data.

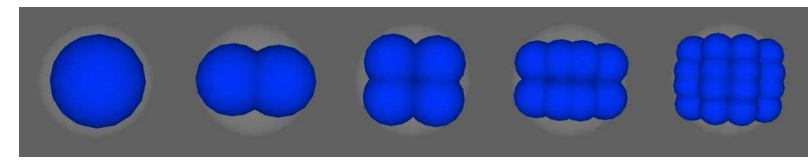

Figure 7. First simulated segmentation of the zebrafish

We also tried to observe spacial constraints when Vitellius is half covered (see Figure 8) to understand the resulting mechanisms (self-adaptation) implemented by cells. And 
those (self-organization mechanisms) leading to form the backbone of the fish.

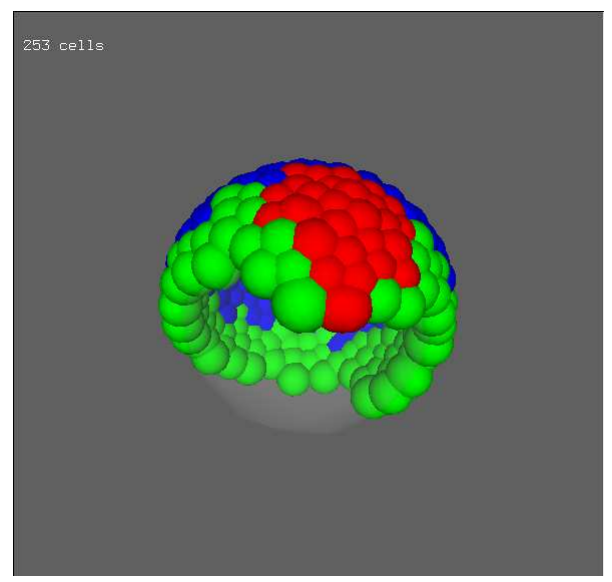

Figure 8. Spaces constraints for the Zebrafish

To better understand morphogenesis, and to overcome obstacles in understanding the influence that the dynamics have on the shape of the organism, we voluntarily limit to discrete simulation by controlling the order of division and ordering executions. Here we have choosen to present a number of simulations by permuting the sequences of mitosis axis, and we observe the impact of these parameters on the shapes. The study is still in its infancy but it is fundamental for understanding the mechanisms controlling morphogenesis.

\section{CONCLUSION}

The main contribution of the paper is the mathematical formalisation of cells behavior leading to a dynamic which create the form (self-organization) and cellular mechanisms ensuring that this dynamic is viable in a set of constraints (self-adaptation). The formalisation relies on the principles of morphological analysis to control cells dynamic and experiment it on a platform of virtual reality. These studies allow us to better understand controls set up by the nature in shapes development.

Among the important issues that have emerged, two issues have attracted particular attention: the robustness of biological forms and the equilibrium of the shape. How cells whose dynamics is simple (mitosis, apoptosis, migration) can maintain their $\mathrm{rm}$ while continually renewing itself (homeostasis), and how despite environmental disruption during embryonic development, the shape stay stable.

\section{REFERENCES}

[1] C. Melani, N. Peyriéras, K. Mikula, C. Zanella, M. Campana, B. Rizzi, F. Veronesi, A. Sarti, B. Lombardot, and P. Bourgine, "Cells tracking in the live zebrafish embryo." in Conf. Proc. IEEE Eng. Med. Biol. Soc., vol. 1, 2007, pp. 1631-1634.
[2] R. Doursat, Organically Grown Architectures: Creating Decentralized, Autonomous Systems by Embryomorphic Engineering. Springer-Verlag, 2008, ch. 8, pp. 167-200, organic computing.

[3] P. Hogeweg and S. Marée, "How amoeboids self-organize into a fruiting body : multicellular coordination in dictyostelium discoideumm," Proc. Natl. Acad. Sci. U.S.A., vol. 98, no. 7, pp. 3879-3883, 2001.

[4] V. Rodin, G. Querrec, P. Ballet, F. Bataille, G. Desmeulles, J. Abgrall, and J. Tisseau, "Multi-agents system to model cell signalling by using fuzzy cognitive maps. application to computer simulation of multiple myeloma." BIBE'09, 9th IEEE International Conference on Bioinformatics and Bioengineering, Taichung (Taiwan), pp. 236-241, 2009.

[5] F. Varela, Principles of biological autonomy, N. Y. Elsevier, Ed., 1979.

[6] J.-P. Aubin, Viability theory. Birkhauser, 1991.

[7] - Mutational and morphological analysis: tools for shape regulation and morphogenesis. Birkhauser, 2000.

[8] T. Lorenz, Mutational Analysis A Joint Framework for Cauchy Problems In and Beyond Vector Spaces. Springer, 2010.

[9] J. Southern, J. Pitt-Francis, J. Whiteley, D. Stokeley, H. Kobashi, R. Nobes, Y. Kadooka, and D. Gavaghan, "Multiscale computational modelling in biology and physiology," Progress in Biophysics and Molecular Biology, vol. 96, no. 9, pp. 60-89, 2008.

[10] P. Reignier, F. Harrouet, S. Morvan, J. Tisseau, and T. Duval, "AReVi: A virtual reality multi-agent platform," Lecture Notes in Computer Science, vol. 1434, pp. 229-240, 1998.

[11] G. Desmeulles, G. Querrec, P. Redou, L. Misery, V. Rodin, and J. Tisseau, "The virtual reality applied to the biology understanding: the in virtuo experimentation," Expert Systems with Applications, Jan. 2006.

[12] B. Lawson and S.Park, "Asynchronous time evolution in an artificial society mode." Journal of Artificial Society and Social Simulation, vol. 3, no. 1, 2000.

[13] P. Chevaillier, S. Bonneaud, G. Desmeulles, and P. Redou, "Experimental study of agent population models with a specific attention to the discretization biases." in In Proceedings of the European Simulation and Modelling Conference (ESM'09), Leicester, UK., 2009, pp. 323-331. 